\title{
Association of Functional VKORC1 Promoter Polymorphism with Occurrence and Clinical Aspects of Ischemic Stroke in a Greek Population
}

\author{
Georgia Ragia, ${ }^{1}$ Stella Marousi, ${ }^{2}$ John Ellul, ${ }^{2}$ Vangelis G. Manolopoulos, ${ }^{1}$ \\ and Anna Tavridou ${ }^{1}$ \\ ${ }^{1}$ Laboratory of Pharmacology, Medical School, Democritus University of Thrace, Dragana Campus, 68100 Alexandroupolis, Greece \\ ${ }^{2}$ Department of Neurology, University of Patras, Rion, 26500 Patras, Greece \\ Correspondence should be addressed to Anna Tavridou; atavrid@med.duth.gr
}

Received 30 June 2013; Revised 25 September 2013; Accepted 9 October 2013

Academic Editor: Ralf Lichtinghagen

Copyright ( 2013 Georgia Ragia et al. This is an open access article distributed under the Creative Commons Attribution License, which permits unrestricted use, distribution, and reproduction in any medium, provided the original work is properly cited.

\begin{abstract}
Genetic factors are considered to play an important role in determining the susceptibility to the occurrence, clinical course, and functional outcome of an acute ischemic stroke (IS). Undercarboxylation of specific vitamin K-dependent proteins, due to genetic polymorphisms of $V K O R C 1$, can affect both vascular calcification and thrombogenicity. We sought to determine the association of VKORC1 -1639G > A polymorphism with IS incidence, age of onset, severity of disease, and functional outcome after an acute IS. VKORC1 -1639G > A polymorphism was determined in 145 consecutive patients with first ever IS and 145 age- and sex-matched control subjects of Greek Caucasian origin using PCR-RFLP. Stroke severity and functional outcome were assessed on admission and at one month after stroke, respectively. Frequency of $V K O R C 1-1639 G>A$ genotypes did not differ between IS patients and controls $(\mathrm{OR}=1.12, P=0.51)$. Moreover, carriage of the $A$ allele was not associated with age of stroke onset, severity of disease (Scandinavian stroke scale score 32.2 versus 32.9, resp., $P=0.96$ ), or poor outcome at 1 month post-stroke ( 52.9 versus $64.4 \%$, resp., $P=0.31$ ). In conclusion, $V K O R C 1-1639 G>A$ polymorphism is not a genetic determinant of IS occurrence, age of onset, severity, or functional outcome of disease in a Greek population.
\end{abstract}

\section{Introduction}

Ischemic stroke (IS), a multifactorial disease which shares many common risk factors with coronary artery disease, leads to a high mortality and disability rate [1]. However, distinct mechanisms may be critical in the development of acute ischemic coronary and cerebrovascular events. Both environmental and genetic factors are considered to play important role in determining the susceptibility to the occurrence, clinical course, and functional outcome of an acute IS. There is evidence that common variants in several genes, each exerting a modest effect, contribute to the risk of stroke [2].

Vitamin K-dependent proteins play a significant role in coagulation but also in bone metabolism and vascular calcification. Modification by $\gamma$-carboxylation is necessary for vitamin K-dependent proteins to become biologically active [3]. Vitamin K epoxide reductase complex subunit 1 (VKORC1) is involved in this process by mediating recycling of vitamin $\mathrm{K}$ 2,3 epoxide to vitamin $\mathrm{K}$ hydroquinone [4], a cofactor for the conversion of glutamate to $\gamma$-carboxyglutamic acid. VKORC1 is the target of coumarin derivatives, and several genetic variations of the VKORCl gene influence response to anticoagulant therapy (for review, see [5]). Matrix Gla protein (MGP), a vitamin K-dependent protein, is important in the prevention of vascular calcification since MGP-deficient mice suffer extensive media calcification of the aorta [6]. Inhibition of VKORC1 by warfarin results in undercarboxylation of MGP and subsequent media calcification in rat arteries and heart valves [7].

Single-nucleotide polymorphisms (SNPs) of VKORC1 have been associated with a higher risk of arterial vascular disease, including stroke, in a Chinese population [8], and a significantly higher risk of aortic calcification [9]. However, two subsequent studies in German cohorts did not 
detect any association between genetic polymorphisms of VKORC1 and either coronary heart disease [10] or stroke [11]. VKORC1 $-1639 G>A$ polymorphism (rs9923231) is located in the promoter of VKORC1 gene. This SNP tags the wide-spread haplotype $V K O R C 1^{*} 2$ [12] and results in lowering mRNA expression, protein, and activity of VKORC1 in subjects with the $A A$ genotype [13]. Consequently, in these subjects, inhibition of vitamin $\mathrm{K}$ recycling would be expected to cause impaired $\gamma$-carboxylation of vitamin $\mathrm{K}$ dependent proteins, such as MGP, and increased vascular calcification. This is supported by a study showing that long-term use of oral vitamin $\mathrm{K}$ antagonists is associated with enhanced extracoronary vascular calcification, possibly through the inhibition of MGP carboxylation [14]. Moreover, we have shown an independent association between VKORC1 $-1639 G>A$ polymorphism and maximum carotid intimamedia thickness (cIMT) in type 2 diabetes mellitus patients [15]. On the other hand, decreased carboxylation of vitamin $\mathrm{K}$-dependent proteins within the arterial vessel wall suggests the presence of less-active coagulation factors (prothrombin, factors VII and X) in human atherosclerotic plaques which determine thrombogenicity [16].

Since the effect of $V K O R C 1$ genetic variants in developing IS is inconclusive and no data exist on their possible role in clinical aspects of disease, our aim was to investigate whether VKORC1 $-1639 G>A$ polymorphism is associated with the risk for IS as well as the age of stroke onset, the severity, and functional outcome after an acute IS.

\section{Subjects and Methods}

2.1. Study Population. A total of 290 unrelated subjects of Greek Caucasian origin were genotyped. Cases presented consecutively at the Emergency Department of the University Hospital of Patras, Patras, Greece, with signs and symptoms of a cerebrovascular event and were subsequently admitted to Neurology and Internal Medicine wards. Patients with intracerebral or subarachnoid hemorrhage, transient ischemic attacks, chronic inflammatory/rheumatic diseases, comorbid malignancy, and strokes secondary to rare conditions (i.e., traumatic carotid dissection and endocarditis) were excluded. All IS patients had a brain computerized tomography (CT) on admission and a second brain imaging (CT or magnetic resonance imaging) during hospitalization. Investigations such as carotid ultrasonography, serology and coagulation studies were performed according to patientspecific clinical criteria. Pre stroke and one month after stroke disability was assessed using the modified Oxford Handicap scale (Rankin) [17]. Good outcome at one month after stroke was defined as Rankin $\leq 1$ and poor outcome as Rankin $\geq 2$ or death. Neurological assessment was carried out using the Scandinavian Stroke Scale (SSS) [18] within 24 hours from stroke onset. Patients were subsequently stratified into three categories of stroke severity: "severe stroke" for scores 0-32, "moderate score" for scores 33-44, and "mild stroke" for scores 45-48 [19]. Classification of stroke subtype was based on TOAST definitions [20]. One hundred and forty five age- and sex-matched control subjects, unrelated to the IS patients, were recruited from routine visits in local community health care facilities. Control subjects with positive IS/transient ischemic attack history, chronic inflammatory/rheumatic disease, and malignancy were excluded. Atrial fibrillation, ischemic heart disease, smoking, arterial hypertension, diabetes mellitus, and hypercholesterolemia were defined as described previously [21]. All subjects participated after being informed about the study and giving written consent. The study protocol was approved by Ethical Committee of the University Hospital of Patras.

\subsection{DNA Isolation and VKORC1 $-1639 G>A$ Genotyping.} Genomic DNA was extracted from whole blood in EDTA, according to standard purification protocols by Qiagen, QIAamp DNA blood Mini kit, and stored at $-80^{\circ} \mathrm{C}$ until use. Genotyping for the VKORC1 $-1639 G>A$ polymorphism was performed by a polymerase chain reaction-restriction fragment length polymorphism (PCR-RFLP) analysis, as described elsewhere in detail [15], in the Laboratory of Pharmacology in the Medical School of Democritus University of Thrace. Genotyping was done in duplicate in all samples. Additionally, $10 \%$ of the samples were randomly selected and genotyped by a different investigator who was blinded as to the outcome of previous analyses. Correct genotype assignment was confirmed in all cases.

2.3. Statistical Analysis. Continuous variables are presented as mean \pm standard deviation (SD) and categorical variables as absolute number and percentage value. Parametric and nonparametric variables were compared by $t$-test and MannWhitney test, respectively. The $\chi^{2}$ test was used to determine whether VKORC1 $-1639 G>A$ genotype distributions and allele frequencies were different between IS patients and controls. Observed genotypes were compared with those expected under Hardy-Weinberg equilibrium (HWE) using the $\chi^{2}$ test in each group. Logistic regression analysis was used to calculate odds ratio (OR) as a measure of association of genotype with IS, under assumptions of additive mode of inheritance, adjusting for established risk factors for IS. For OR, a 2-tailed probability value and 95\% CI were calculated. A Kaplan-Mayer disease-free survival analysis was performed to test for possible differences in the age of IS onset, depending on VKORC1 -1639G $>$ A genotype. Statistical significance was expressed with $P \log$ ranks. A value of $P<0.05$ was considered statistically significant. The Statistical Package for the Social Sciences (SPSS) program (version 16.0) was used for all analyses.

\section{Results}

The anthropometric, clinical, and biochemical characteristics of patients and control subjects included in the study are shown in Table 1 . The two groups did not differ significantly in body mass index (BMI) and smoking habit. However, the prevalence of atrial fibrillation, ischemic heart disease, arterial hypertension, type II diabetes mellitus, and hypercholesterolemia was significantly higher in IS patients compared with controls. 
TABLE 1: Baseline anthropometric and clinical characteristics of subjects included in the study.

\begin{tabular}{lccc}
\hline & $\begin{array}{c}\text { Stroke } \\
(n=145)\end{array}$ & $\begin{array}{c}\text { Controls } \\
(n=145)\end{array}$ & $P$ \\
\hline Sex, females, $n(\%)$ & $50(34.5)$ & $50(34.5)$ & $\mathrm{NS}^{\mathrm{a}}$ \\
$\begin{array}{l}\text { Age (years) } \\
\text { BMI }\left(\mathrm{kg} / \mathrm{m}^{2}\right)\end{array}$ & $67 \pm 12$ & $67 \pm 13$ & $\mathrm{NS}$ \\
$\begin{array}{l}\text { Atrial fibrillation, } n(\%) \\
\begin{array}{l}\text { Ischemic heart disease, } n \\
(\%)\end{array}\end{array}$ & $37.93 \pm 5.09$ & $28.37 \pm 4.17$ & $\mathrm{NS}$ \\
$\begin{array}{l}\text { Current smoking, } n(\%) \\
\begin{array}{l}\text { Arterial hypertension, } n \\
(\%)\end{array}\end{array}$ & $52(36)$ & $81(6)$ & $<0.001$ \\
$\begin{array}{l}\text { Diabetes mellitus, } n(\%) \\
\begin{array}{l}\text { Hypercholesterolemia, } n \\
(\%)\end{array}\end{array}$ & $104(72)$ & $76(52)$ & 0.004 \\
\hline
\end{tabular}

${ }^{a} \mathrm{NS}$ : nonsignificant.

Continuous variables were compared by $t$-test and categorical variables by $\chi^{2}$ test.

In the population as a whole, the frequencies of the genotypes of the $V K O R C 1-1639 G>A$ polymorphism were $32.4 \% G G$ homozygous, $44.8 \% G A$ heterozygous, and $22.8 \%$ $A A$ homozygous. Frequencies of $V K O R C 1-1639 G>A$ genotypes and alleles for IS patients and controls are presented in Table 2. The observed frequencies of genotypes in controls were in $\operatorname{HWE}\left(\chi^{2}=0.064, P=0.8\right)$, whereas for IS patients marginally deviated from $\operatorname{HWE}\left(\chi^{2}=4.074, P=\right.$ 0.04 ). The frequencies of $V K O R C 1-1639 G>A$ genotypes were similar between the two groups either in an additive $(P=0.41)$ or a recessive model $(P=0.33)$. Similar findings were observed from logistic regression analysis which failed to reveal a significant association between phenotype and genotype after adjustment for known risk factors for IS (OR = $1.12, P=0.51)$. Subclassification according to TOAST was performed for the majority of patients. However, we did not find any association between VKORC1 -1639G > $A$ genotypes and any stroke subtype which could be due to the small number of patients in each group (data not shown). Allele frequencies for $G$ and $A$ alleles were $53.8 \%$ and $46.2 \%$ in IS patients and $55.9 \%$ and $44.1 \%$ in controls, respectively, and did not differ significantly between the two groups $(P=0.77)$. Moreover, no differences were found in the frequencies of $V K O R C 1-1639 G>A$ genotypes with respect to atrial fibrillation, ischemic heart disease, hypertension, and hypercholesterolemia (data not shown). In contrast to our previous findings on the association of VKORC1 -1639G > $A$ genotypes with type II diabetes [15], in the population of the present study the frequency of VKORC1 $-1639 G>A$ polymorphism did not differ with respect to type II diabetes mellitus, probably due to the small number of individuals with type II diabetes mellitus $(n=62)$ (data not shown). Due to the high prevalence of hypercholesterolemia and hypertension in IS patients and controls, we also compared the frequencies of $V K O R C 1-1639 G>A$ genotypes between IS
TABLE 2: Frequencies of VKORC1 $-1639 G>A$ genotypes and alleles in IS patients and controls.

\begin{tabular}{|c|c|c|c|c|c|c|c|}
\hline & \multicolumn{3}{|c|}{ IS patients $(n=145)$} & \multicolumn{3}{|c|}{ Controls $(n=145)$} & \multirow[t]{2}{*}{$P$} \\
\hline & $n$ & $\%$ & $95 \% \mathrm{CI}$ & $n$ & $\%$ & $95 \% \mathrm{CI}$ & \\
\hline \multicolumn{8}{|l|}{ Genotypes } \\
\hline$G G$ & 48 & 33.1 & $25.8-41.0$ & 46 & 31.7 & $24.6-39.6$ & \multirow{3}{*}{0.41} \\
\hline$G A$ & 60 & 41.4 & $33.6-49.5$ & 70 & 48.3 & $40.2-56.4$ & \\
\hline$A A$ & 37 & 25.5 & $19.0-33.1$ & 29 & 20.0 & $14.1-27.1$ & \\
\hline$G G / G A$ & 108 & 74.5 & $67.0-81.1$ & 116 & 80.0 & $72.9-85.9$ & \multirow{2}{*}{0.33} \\
\hline$A A$ & 37 & 25.5 & $19.0-33.1$ & 29 & 20.0 & $14.1-27.1$ & \\
\hline \multicolumn{8}{|l|}{ Alleles } \\
\hline$G$ & 156 & 53.8 & $48.0-59.5$ & 162 & 55.9 & $50.1-61.5$ & \multirow{2}{*}{0.77} \\
\hline$A$ & 134 & 46.2 & $40.5-52.0$ & 128 & 44.1 & $38.5-49.9$ & \\
\hline
\end{tabular}

patients and controls stratified by these two clinical variables but did not find a difference (data not shown).

In addition, we sought to explore the association between VKORC1 $-1639 G>A$ polymorphism and stroke severity on admission or functional outcome (Table 3 ). Stroke severity in patients with the $A A$ genotype was similar to the one observed in patients with the GG/GA genotype (SSS score 32.2 versus $32.9, P=0.96)$. Similarly, poor outcome at one month after stroke did not differ significantly between the two groups $(52.9 \%$ versus $64.4 \%, P=0.31)$. Figure 1 shows the Kaplan-Mayer curves for the disease-free survival analysis depending on VKORC1 genotypes according to the recessive model. Carriers of the $A A$ genotype did not differ significantly in the age of stroke onset compared with carriers of the $G G / G A$ genotype (median age in years (standard error), 66 (3.5) versus 68 (2.2), resp., $\log \operatorname{rank} P=0.98)$.

\section{Discussion}

IS represents a complex trait, which is assumed to be polygenic. Therefore, the role of a wide number of candidate genes has been investigated in stroke through association studies. In search of genetic factors that affect IS, we investigated the role of VKORC1 $-1639 G>A$ polymorphism in a Caucasian population. As expected, the prevalence of recognized risk factors for IS was higher in patients compared with controls, but the frequencies of $-1639 G>A$ polymorphism genotypes did not differ with respect to these factors.

There is evidence of ethnic differences in VKORC1 $-1639 G>A$ polymorphism. In Caucasians, the frequency of $A$ allele has been reported to range from 40 to $50 \%$, and our results are in agreement with previous studies [22]. However, in Asian populations the frequency of $A$ allele is approximately $90 \%$.

We did not find a significant difference in the frequencies of $V K O R C 1-1639 G>A$ genotypes or alleles in IS patients when compared to controls. A possible explanation for the lack of association could be the simultaneous effects of VKORC1 -1639 A allele on vitamin K-dependent hemostatic and nonhemostatic proteins, affecting divergently thrombogenicity and calcification. It has been shown that 
TABLE 3: VKORC1 -1639G > A polymorphism in relation to stroke severity on admission and functional outcome at one month after stroke.

\begin{tabular}{lccccccc}
\hline Genotypes & Severe & Moderate & Mild & $P^{*}$ & SSS score mean (SD) & $P^{* *}$ & ${\text { Poor outcome }\left(\mathrm{mRS}^{\mathrm{a}} \geq 2\right)}_{P^{* * *}}$ \\
\hline GG/GA & $33(75.0 \%)$ & $25(75.8 \%)$ & $21(75.0 \%)$ & \multirow{2}{*}{0.99} & $32.9(14.8)$ & 0.96 & $67(64.4 \%)$ \\
$A A$ & $11(25.0 \%)$ & $8(24.2 \%)$ & $7(25.0 \%)$ & & $32.2(15.2)$ & & $18(52.9 \%)$ \\
\hline
\end{tabular}

${ }^{\mathrm{a}} \mathrm{mRS}$ : modified Rankin scale.

$P^{*}$ refers to the distribution of $V K O R C 1$ genotypes within stroke severity groups $\left(\chi^{2}\right.$ test).

$P^{* *}$ refers to the comparison of mean Scandinavian Stroke Scale (SSS) scores between VKORC1 genotypes (Mann-Whitney test).

$P^{* * *}$ refers to the distribution of VKORC1 genotypes within functional outcome groups ( $\chi^{2}$ test).

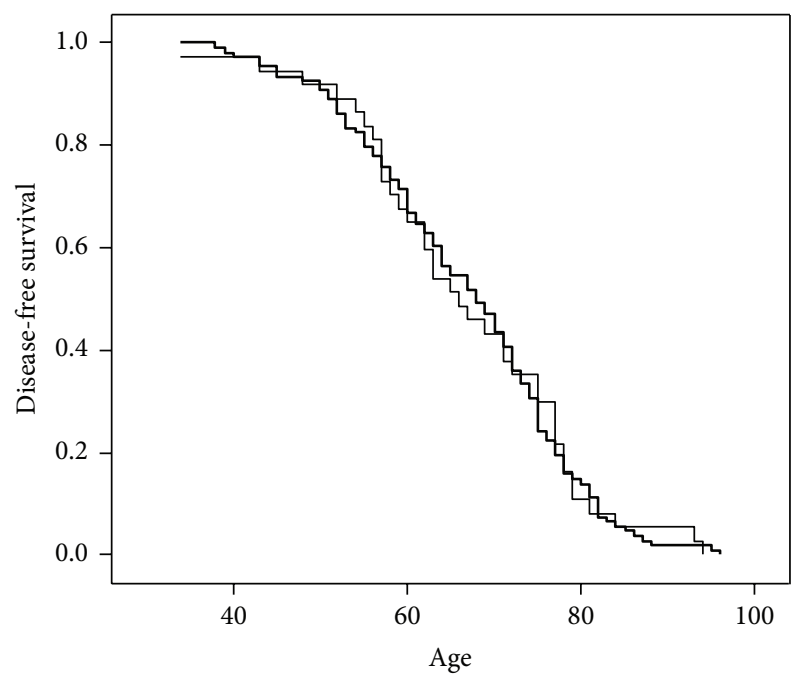

VKORC1 genotype, $\log$ rank $=0.98$ $\neg G G / G A$ $\neg A A$

FIGURE 1: Kaplan-Mayer curve for the disease-free survival analysis according to VKORC1 $-1639 G>A$ polymorphism.

VKORC1 gene polymorphisms per se affect the carboxylation of coagulant factors [23]. However, it should be noted that in cases of mild vitamin K insufficiency the liver is preferentially supplied with vitamin $\mathrm{K}$, so that all vitamin $\mathrm{K}$-dependent coagulation factors are fully carboxylated in the normal adult population [24]. This biological homeostatic process potentially overcomes the effect of $V K O R C 1-1639 G>A$ polymorphism on the coagulation cascade. Furthermore, IS incidence could be the result of anticoagulation failure in atrial fibrillation patients treated with coumarinic oral anticoagulants. Homozygous VKORC1 $-1639 G G$ atrial fibrillation patients treated with coumarinic anticoagulants require higher doses of coumarinic anticoagulants and often need longer time to achieve therapeutic INR [25-28]. Therefore, in VKORC1 $-1639 G G$ patients higher rates of stroke incidence would be expected due to decreased anticoagulation [29], opposing the possible effect of $-1639 \mathrm{~A}$ allele on stroke incidence through carboxylation of vitamin K-dependent proteins. In the present study, the incidence of atrial fibrillation was significantly higher in IS patients than in controls $(21 \%$ versus 6\%); however, the low number of VKORC1 -1639GG homozygous atrial fibrillation patients $(n=17)$ allows us to exclude the possibility of therapeutic failure masking the effect of $A$ allele. An alternative explanation for the lack of association may be that MGP is a strong inhibitor of media calcification, whereas its possible effect on calcification of atherosclerotic lesions and plaque remains a matter of dispute. Hitherto, an association between media calcification and IS has not been shown. Moreover, it would be interesting to assess the effect of VKORC1-1639G $>A$ polymorphism on MGP carboxylation by measuring the uncarboxylated form of the protein in plasma. Another potential explanation of the lack of effect of $V K O R C 1-1639 G>A$ polymorphism on IS could be that most of the risk of disease can be attributed to the high prevalence of known risk factors such as hypertension and hypercholesterolemia in our cohort.

Only few studies have investigated the association for VKORC1 genetic polymorphisms with the risk for developing an IS. In a Chinese population, the ancestral $C$ allele of VKORC1 $+2255 T / C$ polymorphism (rs2359612), which is in nearly complete linkage disequilibrium with VKORC1 $-1639 G>A$ polymorphism, is the minor allele and more frequent in stroke patients than in controls [8]. Although the mechanism behind this finding remains unclear, it could be due to the contribution of increased $\gamma$-glutamyl carboxylation of blood coagulation proteins to the progression and thrombogenicity of atherosclerotic lesions as well as a potential need for increased warfarin dosage. However, in a later study, Shyu et al. did not find an association between VKORC1 $-1639 G>A$ polymorphism and stroke in a Chinese population, despite similar allele frequencies [30]. On the contrary, in Caucasians, the frequency of VKORC1 -1639G allele is higher compared with Asian populations and the major one observed. Therefore, its association with cardiovascular disease cannot be anticipated by studies in Asian populations. Two studies performed in German populations did not find an association between VKORC1 -1639G > A polymorphism and either coronary heart disease or stroke $[10,11]$. Our findings are in agreement with the latter study, despite differences in the frequency of known risk factors for stroke between the two studies.

Given the possible diverse pathophysiological roles of VKORC1 in vascular disease and its effect on response to coumarinic anticoagulants, we also sought to determine whether VKORC1 $-1639 G>A$ polymorphism could be associated with age of onset, severity, and adverse outcome of IS, not addressed by previous studies. However, we were not able to demonstrate any association of VKORC1 $-1639 G>A$ polymorphism with the studied clinical aspects of disease.

This case-control study has some limitations, as the study group is quite small and this might lead to nonrepresentative 
results. Most genetic association studies use an inadequate number of cases and controls and, therefore, lack the ability to draw firm conclusions on the observed associations. However, they are still useful since they can be used in future meta-analysis which can summarize such data and make a conclusion on the associations. The high frequency of established risk factors for stroke, such as hypertension, diabetes mellitus, or hypercholesterolemia, may obscure the association between $V K O R C 1-1639 G>A$ polymorphism and IS. Other unknown functional polymorphisms may also influence the expression of VKORCl and their combined effect must be studied to better predict the occurrence, severity, and outcome of IS.

\section{Conclusions}

In the present study, VKORC1 $-1639 G>A$ polymorphism was not associated with the occurrence, age of onset, severity, and outcome of IS in a Caucasian population. However, the lack of association between this polymorphism and IS does not exclude the existence of other variants in this gene or its interaction with other genes that could influence the risk of developing IS. More research, including different ethnic groups, is necessary to make a conclusion on the association between VKORC1 -1639G > A polymorphism and IS.

\section{References}

[1] A. S. Go, D. Mozaffarian, V. L. Roger et al., "Heart disease and stroke statistics-2013 update: a report from the American Heart Association," Circulation, vol. 127, no. 1, pp. e6-e245, 2013.

[2] J. P. Casas, A. D. Hingorani, L. E. Bautista, and P. Sharma, "Meta-analysis of genetic studies in ischemic stroke: thirtytwo genes involving approximately 18,000 cases and 58,000 controls," Archives of Neurology, vol. 61, no. 11, pp. 1652-1661, 2004.

[3] K. L. Berkner, "Vitamin K-dependent carboxylation," Vitamins and Hormones, vol. 78, pp. 131-156, 2008.

[4] T. Li, C.-Y. Chang, D.-Y. Jin, P.-J. Lin, A. Khvorova, and D. W. Stafford, "Identification of the gene for vitamin K epoxide reductase," Nature, vol. 427, no. 6974, pp. 541-544, 2004.

[5] V. G. Manolopoulos, G. Ragia, and A. Tavridou, "Pharmacogenetics of coumarinic oral anticoagulants," Pharmacogenomics, vol. 11, no. 4, pp. 493-496, 2010.

[6] G. Luo, P. Ducy, M. D. McKee et al., "Spontaneous calcification of arteries and cartilage in mice lacking matrix GLA protien," Nature, vol. 386, no. 6620, pp. 78-81, 1997.

[7] P. A. Price, S. A. Faus, and M. K. Williamson, "Warfarin causes rapid calcification of the elastic lamellae in rat arteries and heart valves," Arteriosclerosis, Thrombosis, and Vascular Biology, vol. 18, no. 9, pp. 1400-1407, 1998 .

[8] Y. Wang, W. Zhang, Y. Zhang et al., "VKORC1 haplotypes are associated with arterial vascular diseases (stroke, coronary heart disease, and aortic dissection)," Circulation, vol. 113, no. 12, pp. 1615-1621, 2006.

[9] M. Teichert, L. E. Visser, R. H. N. Van Schaik et al., "Vitamin K epoxide reductase complex subunit 1 (VKORC1) polymorphism and aortic calcification: the Rotterdam study," Arteriosclerosis, Thrombosis, and Vascular Biology, vol. 28, no. 4, pp. 771-776, 2008.
[10] M. Watzka, A. Nebel, N. E. El Mokhtari et al., "Functional promoter polymorphism in the VKORC1 gene is no major genetic determinant for coronary heart disease in Northern Germans," Thrombosis and Haemostasis, vol. 97, no. 6, pp. 9981002, 2007.

[11] M.-L. Arnold, C. Lichy, I. Werner, A. Radbruch, S. Wagner, and C. Grond-Ginsbach, "Single nucleotide polymorphisms in the VKORCl gene and the risk of stroke in the Southern German population," Thrombosis and Haemostasis, vol. 100, no. 4, pp. 614-617, 2008.

[12] C. Geisen, M. Watzka, K. Sittinger et al., "VKORC1 haplotypes and their impact on the inter-individual and inter-ethnical variability of oral anticoagulation," Thrombosis and Haemostasis, vol. 94, no. 4, pp. 773-779, 2005.

[13] H.-Y. Yuan, J.-J. Chen, M. T. M. Lee et al., "A novel functional VKORC1 promoter polymorphism is associated with interindividual and inter-ethnic differences in warfarin sensitivity," Human Molecular Genetics, vol. 14, no. 13, pp. 1745-1751, 2005.

[14] R. J. M. W. Rennenberg, B. J. Van Varik, L. J. Schurgers et al., "Chronic coumarin treatment is associated with increased extracoronary arterial calcification in humans," Blood, vol. 115, no. 24, pp. 5121-5123, 2010.

[15] A. Tavridou, I. Petridis, M. Vasileiadis et al., "Association of VKORC1 $-1639 G>A$ polymorphism with carotid intimamedia thickness in type 2 diabetes mellitus," Diabetes Research and Clinical Practice, vol. 94, no. 2, pp. 236-241, 2011.

[16] H. M. H. Spronk, "Vitamin K epoxide reductase complex and vascular calcification: is this the important link between vitamin $\mathrm{K}$ and the arterial vessel wall?" Circulation, vol. 113, no. 12, pp. 1550-1552, 2006.

[17] J. M. Bamford, P. A. Sandercock, C. P. Warlow, and J. Slattery, "Interobserver agreement for the assessment of handicap in stroke patients," Stroke, vol. 20, no. 6, p. 828, 1989.

[18] E. Aberg, G. Adielsson, A. Almqvist et al., "Multicenter trial of hemodilution in acute ischemic stroke. Results of subgroup analyses," Stroke, vol. 19, no. 4, pp. 464-471, 1988.

[19] G. Gioldasis, P. Talelli, E. Chroni, J. Daouli, T. Papapetropoulos, and J. Ellul, "In-hospital direct cost of acute ischemic and hemorrhagic stroke in Greece," Acta Neurologica Scandinavica, vol. 118, no. 4, pp. 268-274, 2008.

[20] H. P. Adams Jr., B. H. Bendixen, L. J. Kappelle et al., "Classification of subtype of acute ischemic stroke: definitions for use in a multicenter clinical trial," Stroke, vol. 24, no. 1, pp. 35-41, 1993.

[21] S. G. Marousi, J. Ellul, A. Antonacopoulou, C. Gogos, P. Papathanasopoulos, and M. Karakantza, "Functional polymorphisms of interleukin 4 and interleukin 10 may predict evolution and functional outcome of an ischaemic stroke," European Journal of Neurology, vol. 18, no. 4, pp. 637-643, 2011.

[22] K. A. Ross, A. W. Bigham, M. Edwards, A. Gozdzik, G. Suarez-Kurtz, and E. J. Parra, "Worldwide allele frequency distribution of four polymorphisms associated with warfarin dose requirements," Journal of Human Genetics, vol. 55, no. 9, pp. 582-589, 2010.

[23] R. Kimura, Y. Kokubo, K. Miyashita et al., "Polymorphisms in vitamin $\mathrm{K}$-dependent $\gamma$-carboxylation-related genes influence interindividual variability in plasma protein $C$ and protein $S$ activities in the general population," International Journal of Hematology, vol. 84, no. 5, pp. 387-397, 2006.

[24] E. Theuwissen, E. Smit, and C. Vermeer, "The role of vitamin K in soft-tissue calcification," Advances in Nutrition, vol. 3, no. 2, pp. 166-173, 2012. 
[25] R. C. Johnson, J. A. Leopold, and J. Loscalzo, "Vascular calcification: pathobiological mechanisms and clinical implications," Circulation Research, vol. 99, no. 10, pp. 1044-1059, 2006.

[26] S. S. Rathore, S. K. Agarwal, S. Pande, S. K. Singh, T. Mittal, and B. Mittal, "Pharmacogenetic aspects of coumarinic oral anticoagulant therapies," Indian Journal of Clinical Biochemistry, vol. 26, no. 3, pp. 222-229, 2011.

[27] E. A. Sconce, T. I. Khan, H. A. Wynne et al., "The impact of CYP2C9 and VKORC1 genetic polymorphism and patient characteristics upon warfarin dose requirements: proposal for a new dosing regimen," Blood, vol. 106, no. 7, pp. 2329-2333, 2005.

[28] E. Stepień, A. Branicka, M. Cieśla-Dul, and A. Undas, "A vitamin K epoxide reductase-oxidase complex gene polymorphism $(-1639 G>A)$ and interindividual variability in the dose-effect of vitamin K antagonists," Journal of Applied Genetics, vol. 50, no. 4, pp. 399-403, 2009.

[29] L. M. Brass, H. M. Krumholz, J. M. Scinto, and M. Radford, "Warfarin use among patients with atrial fibrillation," Stroke, vol. 28, no. 12, pp. 2382-2389, 1997.

[30] H.-Y. Shyu, C.-S. Fong, Y.-P. Fu et al., "Genotype polymorphisms of GGCX, NQO1, and VKORC1 genes associated with risk susceptibility in patients with large-artery atherosclerotic stroke," Clinica Chimica Acta, vol. 411, no. 11-12, pp. 840-845, 2010 . 


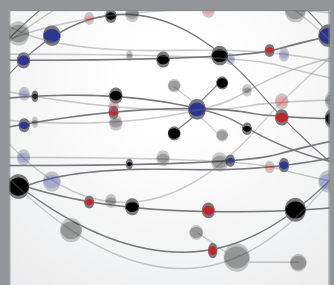

The Scientific World Journal
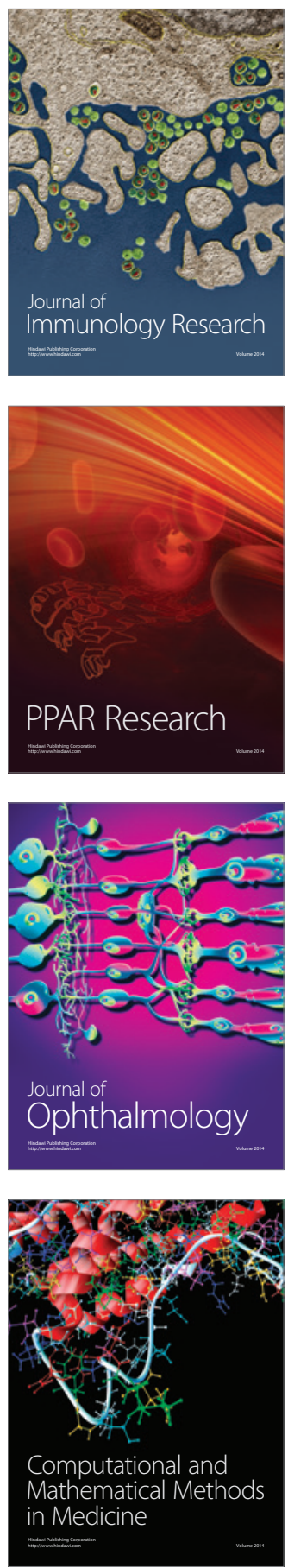

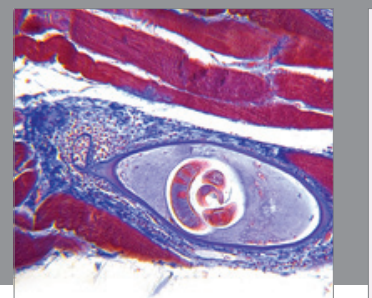

Gastroenterology

Research and Practice
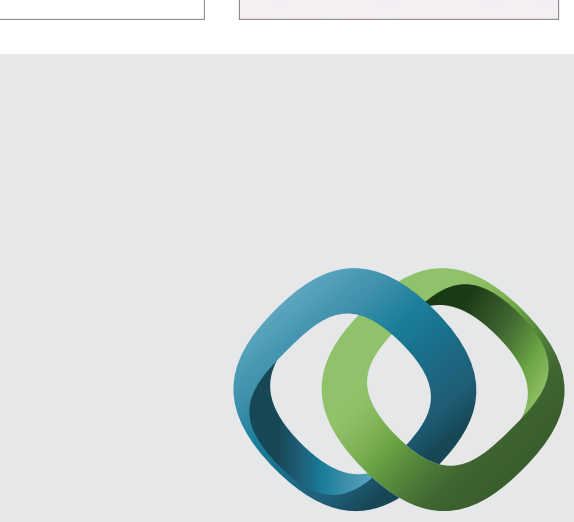

\section{Hindawi}

Submit your manuscripts at

http://www.hindawi.com
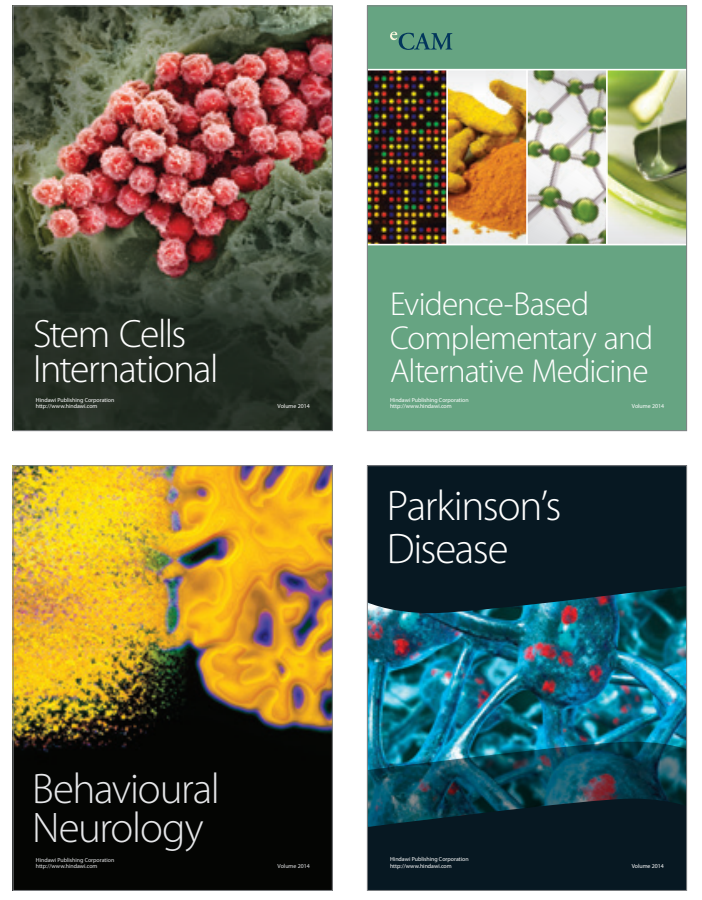
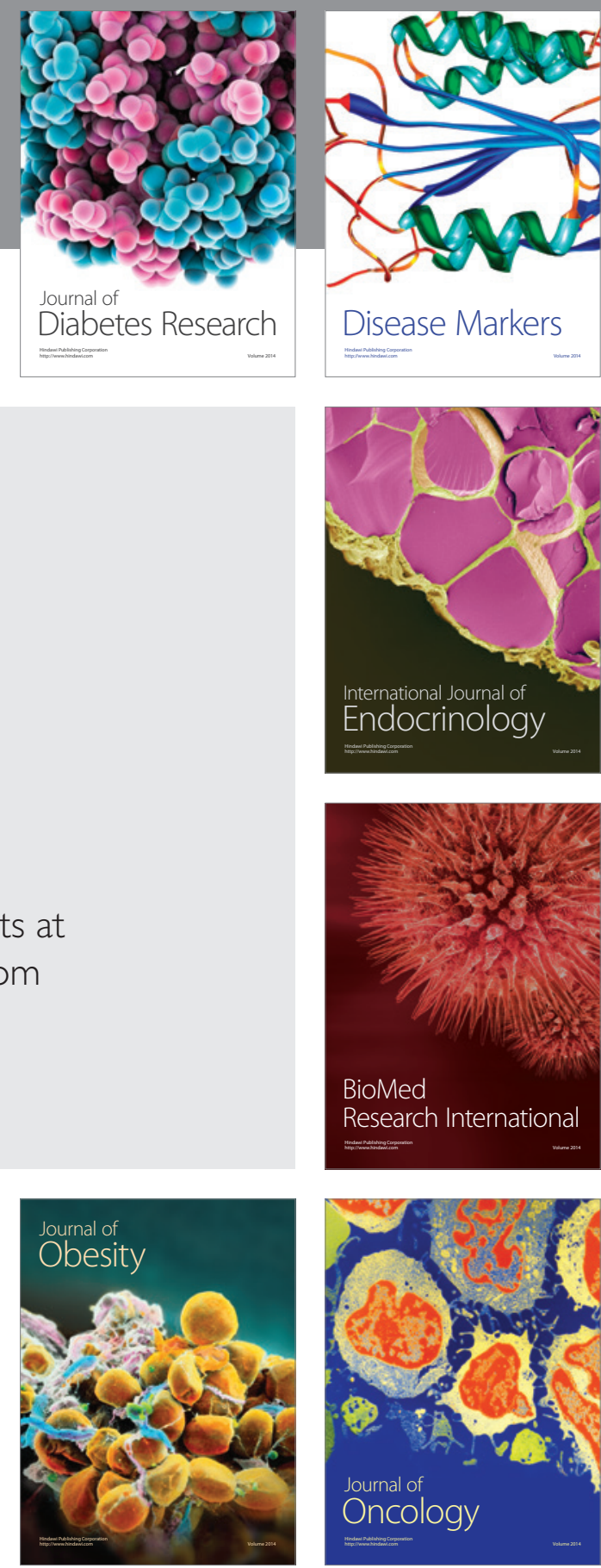

Disease Markers
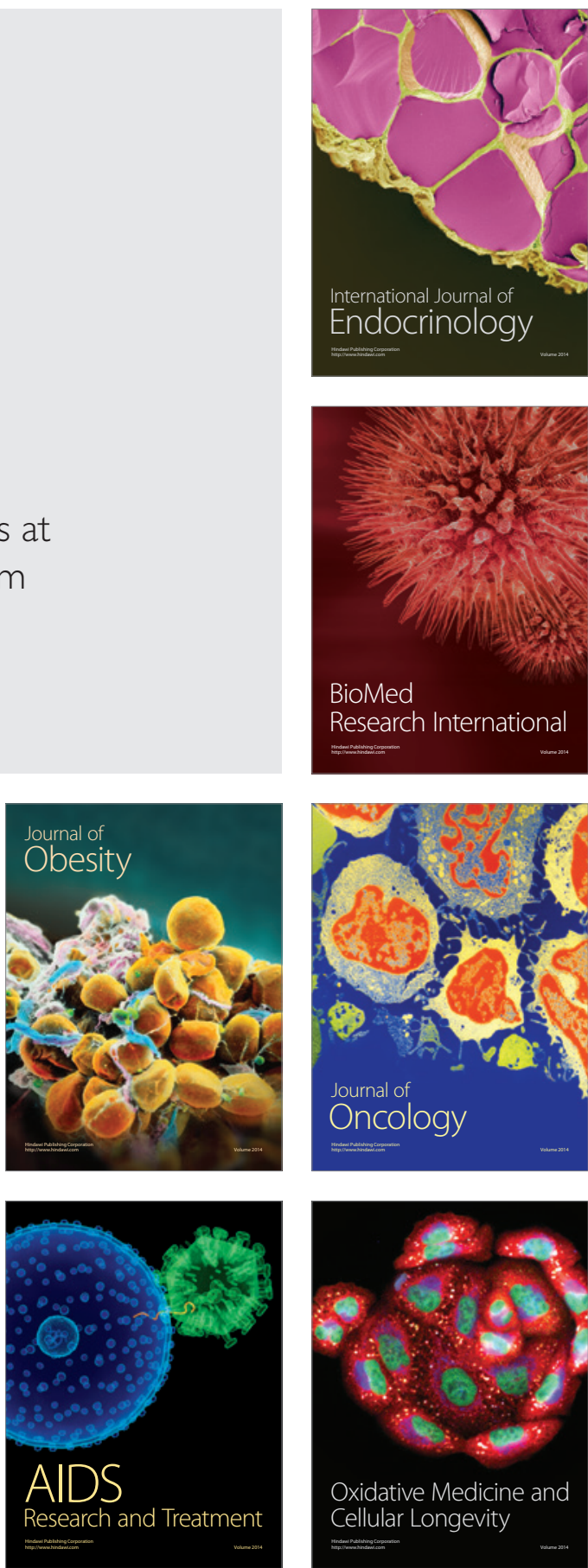